\title{
CALIDAD DE VIDA RELACIONADA CON LA SALUD: ASPECTOS CONCEPTUALES
}

\author{
HEALTH -RELATED QUALITY OF LIFE: CONCEPTUAL ASPECTS
}

\author{
LAURA SCHWARTZMANN*
}

\begin{abstract}
RESU MEN
Se revisa la importancia del concepto de calidad de vida relacionado con la salud (CVRS) como un aporte fundamental en la evaluación deresultados en salud, al habersetornado insuficientes las medidas tradicionales de morbimortalidad y expectativa de vida. Se destaca la importancia de tomar en cuenta la percepción del paciente, sus deseos y motivaciones en el proceso de toma de decisiones en salud así como en la evaluación de la calidad de la asistencia sanitaria. Se discuten los aspectos conceptuales del constructo "CVRS", sus alcances y sus limitaciones, así como su desarrollo histórico a partir de tres tradiciones básicas, la investigación del concepto defelicidad, en la psicología, la de indicadores sociales en la sociología y la investigación del estado de salud en las ciencias médicas. Se señala asimismo la relatividad histórico-cultural del concepto así como la variabilidad de las prioridades en distintos momentos de la vida. Se exponefinal menteel marco conceptual desdeel quetrabajamosen el Departamento dePsicología M édica de la Facultad deM edicina de la Universidad de la República Oriental del U ruguay así como un modelo conceptual, que debería valorarse empíricamente permitiendo el diseño científico de intervenciones destinadas a promover el bienestar psicosocial, mejorando la calidad de vida de los pacientes.
\end{abstract}

Palabras claves: Calidad de vida relacionado con salud, fuentes conceptuales, valoración de la salud, resultados de la salud, calidad del cuidado.

\begin{abstract}
The article reviews the importance of the concept of health-related quality of life (HRQL) as outcome in health evaluation. Traditional measures of morbidity, mortality and life expectancy are no longer sufficient to determine differences. The need of taking into consideration patients' perceptions, preferences and motivations in decisionmaking and health evaluation is emphasized Conceptual issues on the HRQL construct are reviewed, its strengths and limitations. Historical development of the concept coming from three basic traditions: psychological ( study of happiness), sociological (social indicators) and medical sciences (health status profiles) is revised.

TheH RQ L concept is recognized as individual, age- dependent but also culturally and historically dependent one. Finally the conceptual framework we adhere to at the M edical Psychology Department of the School of medicine (University of the Republic of U ruguay) is developed as well as a proposal for empirical testing allowing scientificallybased psychosocial interventions.
\end{abstract}

Keywords: Health-related quality of life, conceptual issues, health evaluation, health outcomes, quality of care.

Recepcionado: 20.11.2003. Aceptado: 28.12.2003.

"El oficio dela medicina no es sino tocar la curiosa arpa quees el cuerpo humano y restituirlesu armonía".

Francis BACon

\section{INTRODUCCIÓN}

Si bien el tema de la calidad de vida o de la "buena vida" está presente desde la época de los antiguos griegos (Aristóteles), la instalación del concepto dentro del campo dela salud es relativamente reciente, con un auge evidente en la década de los 90, que se man-

* M édico psiquiatra. Profesora Agregada de Psicología M édica de la Facultad de Medicina, Universidad de la Reública O riental del Uruguay. M iembro del Consejo Directivo de la International Society of Life Research (ISO QOL). Correspondencia: Calabria 3969, M ontevideo, CP 11600, Uruguay. E-mail: lauras@mednet.org.uy 
tiene en estos primeros años del siglo XXI. Anualmente se publican en revistas médicas más de 2.000 artículos, poniendo de manifiesto tanto el gran interés como la amplia gama de acepciones del término.

Por otra parte, la vida actual, caracterizada por un aumento en la longevidad, no está necesariamente asociada a mejor calidad de vida. El aumento de la frecuencia y velocidad de los cambios (revolución tecnológica), la inseguridad constante, el exceso de información, el desempleo o el multiempleo, los cambios en la estructura familiar (divorcios, uniones inestables, ambos padres en el mercado laboral), la pérdida de motivaciones, lealtades, valores, señalan los múltiples factores estresantes a que estamos sometidas la mayoríadelossereshumanos. Seconocequeel estrés predisponea la enfermedad y deteriora la calidad de vida. El Grupo de Trabajo Europeo dela Organización M undial dela Salud (Levi, L., 2001) estima para el 2020 que el stress sea la causa principal demuerte, vinculándola en primer lugar a afecciones cardiovasculares y a las depresiones con su consecuente riesgo suicida. Esta situación en queconviven el permanente avance de la ciencia, la enorme producción de bienes, grandes gastos en salud junto a montos elevados de stress y enfermedades asociadas, insatisfacción en gran parte de los usuarios de los servicios de salud, lleva a cuestionarse qué pasa en nuestras sociedades, en general, y en particular qué concepto de salud y definido por quién estamos usando.

Entramos en el siglo XXI, la tecnología avanza a pasos agigantados y la medicina no es ajena a este crecimiento. Los conocimientos disponibles permitirían solucionar los problemas dealimentación dela humanidad. Sin embargo, como señaló el Encuentro Continental de Educación M édica (1994) en la Declaración de Uruguay - en una afirmación que a casi 10 años mantiene total vigencia-, "las significativas transformaciones políticas y económicas y especialmente la reforma del sector salud, iniciada en la mayoría de los países del continente, no se han reflejado positivamente en el desarrollo de las condiciones de vida, de modo de promover con equidad mejoras en las condiciones de salud de nuestras poblaciones".

En relación a la atención en salud, el excesivo énfasis en los aspectos tecnológicos y el deterioro de la comunicación equipo de salud-paciente ha ido quitándole a la relación de ayuda profesional la calidad relacional que fuese otrora soporte social para el paciente y fuentedegratificación y reconocimiento para el profesional de la salud. El diagnóstico y el tratamiento de la enfermedad, a nivel biomé dico exclusivamente, el uso de complicados procedimientos tecnológicos, que sin duda han representado un salto cualitativo a nivel de la supervivencia en enfermedades antes rápidamentemortales, han dejado delado, en muchas ocasiones, la aproximación más holística al cuidado de la salud, donde no sólo se busque combatir la enfermedad sino promover el bienestar.

La prevalencia de enfermedades crónicas, en la mayoría de los países, para las cuales no existe una curación total y donde el objetivo del tratamiento es atenuar o eliminar síntomas, evitar complicaciones y mejorar el bienestar de los pacientes, lleva a que las medidas clásicas de resultados en medicina (mortalidad, morbilidad, expectativa devida) no sean suficientes para evaluar la calidad de los servicios de salud.

En este contexto, la incorporación de la medida dela Calidad deVida Relacionadacon la Salud(CVRS) como una medida necesaria ha sido una de las mayores innovaciones en las evaluaciones (Guyatt, G.H .; Feeny, D.H., Patrick, D., 1993). 


\section{2. ¿QUÉ ES LO NUEVO QUE APORTA A LAS CIENCIASDE LA SALUD EL CON- CEPTO DE CVRS?}

Esencialmenteincorporala percepción del paciente, como una necesidad en la evaluación de resultados en salud, debiendo para ello desarrollar los instrumentos necesarios para que esa medida sea válida y confiable y aporte evidencia empírica con base científica al proceso de toma de decisiones en salud (Testa, M., 1996).

La aplicación, a veces indiscriminada de nuevas tecnologías, con capacidad deprolongar la vida a cualquier precio, la complicada decisión de cantidad versus calidad dela vida, y el terribledilema ético de la distribución de los recursos económicos en salud, ponen sobre el tapete la necesidad de conocer las opiniones de los pacientes. (¿Se siguen haciendo tratamientos pretendidamentecurativosa un paciente con cáncer, fuera de estas posibilidades y aunque los efectos secundarios superen ampliamente los beneficios y deterioren la calidad devida del paciente? ¿A quién compete esa decisión?).

El modelo biomédico tradicional excluye el hecho de que, en la mayoría de las enfermedades, el estado de salud está profundamente influido por el estado de ánimo, los mecanismos de afrontamiento a las diversas situaciones y el soportesocial. Es evidenteque estos aspectos de máxima importancia en la vida de los seres humanos serán los que más influyan a la hora dequelos pacientes evalúen su calidad de vida.

\section{3. ¿POR QUÉ UTILIZAR LAS MEDIDAS DE CVRS?}

1. La toma de decisiones en el sector salud debería tener en cuenta la percepción de los usuarios, apoyada en una profunda evidencia empírica de base científica, que considere, además de los indicadores clásicos cuantitativos (mortalidad, morbilidad, expectativa de vida) y los costos, los indicadores cualitativos que expresan el impacto sobre la calidad de vida y la satisfacción del paciente.

2. Se debería además analizar el proceso de atención a la salud tanto en lo que hace a la evaluación dela excelencia técnica (decisiones terapéuticas, utilización detecnologías de diagnóstico y tratamiento con fundamento sólido) así como la evaluación dela excelencia interpersonal, basada en un manejo científico, ético y humano de la relación médico-paciente.

3. El estudio de los factores que determinan la percepción del paciente en los diversos momentos de la vida y de la enfermedad, es decir el proceso de adaptación a la enfermedad crónica, permitiría reconocer los mecanismosqueinciden negativamenteen la CVRS del paciente y encarar intervenciones psicosociales que promuevan el mayor bienestar posible.

4. Las decisiones exclusivas en base a costos, que se vienen dando en muchos países, donde la medicina pasa a ser una mercancía, son éticamenteinaceptables y desdelos profesionales de la salud debería insistirse en la necesidad detomar en cuenta los otros elementos planteados.

¿Cuánto cuesta un paciente mal atendido, qué impacto tiene el abandono o el incumplimiento de la insulina en un diabético juvenil que no se sintió comprendido por su médico en las dificultades de adaptación a una enfermedad crónica en plena adolescencia?

El esquema de Donabedian (1966) para la evaluación en salud incorporajustamenteestos distintos aspectos. A su propuesta hemos agregado la adaptación del paciente ante la enfermedad en las variables de proceso y la valoración familiar de la "sobrecarga" que la enfermedad implica, como variable de resultados. 
Tabla 1. Evaluación en salud.

\begin{tabular}{|l|l|l|}
\hline \multicolumn{1}{|c|}{ Estructura } & \multicolumn{1}{|c|}{ Proceso } & \multicolumn{1}{c|}{ Resultados } \\
\hline Características de & Excelencia técnica & Situación clínica \\
la comunidad & -Tecnología apropiada & - M orbilidad \\
Características de & -Conocimiento científico & -M ortalidad \\
la organización sanitaria & Excelencia interpersonal & Calidad de vida \\
Características de & -Relación M P & Satisfacción con \\
los proveedores de salud & -Eficiencia & la atención en salud \\
Características de & Evaluación del proceso & Sobrecarga familiar \\
la población & de adaptación del paciente & Expectativa de vida \\
& $\begin{array}{l}\text { a su enfermedad y de sus } \\
\text { determinantes }\end{array}$ & Costos \\
\hline
\end{tabular}

Desde comienzos de la última década del siglo pasado, el estudio de la Calidad deVida Relacionada con la Salud (CVRS) ha logrado captar la atención de muchos investigadores que intentan dar respuesta científica a la necesidad de incluir en las evaluaciones en salud la percepción de los usuarios respecto de su bienestar.

Tradicionalmente, en medicina, se consideraban válidas sólo las observaciones del equipo médico (datos"objetivos"). Posteriormente tomó mayor importancia la consideración de los datos provenientes del paciente (datos "subjetivos"). En la actualidad se in- tenta dejar de lado el debate "objetivo" versus "subjetivo" revalorizándose los datos subjetivos que reflejan sentimientos y percepciones legítimas del paciente que condicionan su bienestar o malestar y su estilo de vida.

Barbara Dickey (Dickey y Wagenar, 1966) propuso cambiar el enfoque objetivo versus subjetivo, hacia el enfoque "informacional". Éste supone atribuir igual importancia a la observación dela enfermedad ("disease") he cha por el clínico, al reportedel pacienteacerca de su padecimiento ("illness") y a la percepción familiar de la sobrecarga ("burden") que la situación implica.

Tabla 2. Perspectivas en salud.

\begin{tabular}{|c|c|c|c|}
\hline Dominio & $\begin{array}{l}\text { Enfermar } \\
\text { "Disease" } \\
\text { Equipo de salud }\end{array}$ & $\begin{array}{l}\text { Padecer } \\
\text { "Illness" } \\
\text { Paciente } \\
\text { Calidad de vida }\end{array}$ & $\begin{array}{l}\text { Soportar } \\
\text { "Burden" } \\
\text { Familia }\end{array}$ \\
\hline Físico & $\begin{array}{l}\text { Morbilidad } \\
\text { M ortalidad }\end{array}$ & $\begin{array}{l}\text { Funcionamiento } \\
\text { Percibido } \\
\text { Dolor }\end{array}$ & $\begin{array}{l}\text { Enfermedades } \\
\text { Familiares por } \\
\text { Stress }\end{array}$ \\
\hline M ental & $\begin{array}{l}\text { Síntomas y signos } \\
\text { neuro-psiquiátricos }\end{array}$ & $\begin{array}{l}\text { Sentimientos } \\
\text { Estados de } \\
\text { ánimo }\end{array}$ & $\begin{array}{l}\text { Sentimientos } \\
\text { Preocupaciones } \\
\text { defuturo }\end{array}$ \\
\hline Social & $\begin{array}{l}\text { Red social } \\
\text { Funcionamiento } \\
\text { en roles asignados }\end{array}$ & $\begin{array}{l}\text { Soporte social } \\
\text { Satisfacción con } \\
\text { los roles o cambios de rol }\end{array}$ & $\begin{array}{l}\text { Soporte social } \\
\text { Satisfacción con los roles } \\
\text { o cambios de rol }\end{array}$ \\
\hline $\begin{array}{l}\text { Salud } \\
\text { general }\end{array}$ & $\begin{array}{l}\text { Severidad de la } \\
\text { enfermedad } \\
\text { Nivel de salud }\end{array}$ & $\begin{array}{l}\text { Salud percibida } \\
\text { Necesidad } \\
\text { de servicios sanitarios }\end{array}$ & $\begin{array}{l}\text { Salud percibida } \\
\text { Necesidad de servicios } \\
\text { sanitarios }\end{array}$ \\
\hline
\end{tabular}




\section{4. ¿QUÉ SE ENTIENDE POR CALIDAD DE VIDA RELACIONADA CON LA SA- LUD?ALCANCES, LIMITACIONESY CONFUSIONES CONCEPTUALES}

La evaluación de calidad de vida en un pacienterepresenta el impacto queuna enfermedad y su consecuente tratamiento tienen sobre la percepción del paciente de su bienestar. Patrick y Erickson (1993) la definen como la medida en que se modifica el valor asignado a la duración dela vida en función dela percepción de limitaciones físicas, psicológicas, sociales y de disminución de oportunidades a causa de la enfermedad, sus secuelas, el tratamiento y/o las políticas de salud.

Para Schumaker \& Naughton (1996) es la percepción subjetiva, influenciada por el estado de salud actual, de la capacidad para realizar aquellas actividades importantes para el individuo.

La esencia de este concepto está en reconocer quela percepción delas personas sobre su estado de bienestar físico, psíquico, social y espiritual depende en gran parte de sus propios valores y creencias, su contexto cultural ehistoria personal.

Si bien la incorporación de las medidas de Calidad deVida Relacionada con la Salud representa uno de los avances más importantes en materia de evaluaciones en salud, no existe aún la claridad suficiente respecto a una base conceptual compartida. El concepto de calidad de vida se ha banalizado en grado extremo, en especial en los campos de la comunicación y del consumo. Por otra parte, es inevitable tener que aceptar la dificultad de poder medir integralmente un fenómeno tan multicausal como es la autoevaluación de la percepción individual, tratando de generar una base empírica, que permita pasar de un discurso genérico einconmensurable a datos que provean evidencia científica de adecuada calidad.

Implica además aceptar que, hasta el momento, las evaluaciones de CVRS asumen que las personas son capaces de analizar aspectos de su estado de salud en forma aislada, separándolos deotros aspectos de la vida humana (ingresos, situación laboral, relaciones interpersonales, estrategias personales de afrontamiento). Hay numerosas evidencias de que, a medida que la enfermedad progresa, ocurren ajustes internos que preservan la satisfacción que la persona siente con la vida, por lo que podemos encontrar personas con grados importantes de limitación física que consideren que su calidad de vida es buena (Leplège y Hunt, 1998).

La confusión entre estado de salud y calidad de vida ha dado origen a dilemas éticos, técnicos y conceptuales. Algunos críticos del concepto han considerado queel mismo conlleva a la medicalización de la vida cotidiana. El concepto de calidad de vida no puede ser de ningún modo independiente de las normas culturales, patrones de conducta y expectativas de cada uno. Sin embargo es frecuente quelas investigaciones deCVRS dejen delado estos aspectos antropológicos y culturales, asumiendo un sistema único globalizado de valores. Pacientes asmáticos estudiados en distintas etapas de su enfermedad referían, independientemente del estadio de su enfermedad, que hechos como pasarlo bien en la vida, experimentar el dar y recibir amor, tener una actitud positiva antelos sucesos dela vida cotidiana eran los factores que proporcionaban a la vida su más alto grado de calidad.

Podría acordarse, por tanto, que es también tarea de la medicina optimizar las situaciones en las quees posible conseguir una calidad devida razonable, incluyen do la promoción de bienestar a través de intervenciones psicosociales, cuando sea necesario, dentro de los tratamientos habituales.

Observamos que la definición propuesta en 1993 deja de lado, al menos en forma explícita, la palabra "bienestar" como parte de la definición. Sin embargo, en la medida en que éste es un campo dinámico, de reciente desarrollo, comienzan a aparecer otros conceptos, no necesariamente compartidos por todos los investigadores, pero que tienen su 
anclaje en la definición de salud de la Organización M undial delaSalud (OMS) de1948: “... un estado de completo bienestar físico, psíquico y social y no meramente la ausencia de enfermedad".

Es claro que calidad de vida es una noción eminentementehumana queserelacionacon el grado de satisfacción quetienela persona con su situación física, su estado emocional, su vida familiar, amorosa, social así como el sentido que le atribuye a su vida, entre otras cosas.

Uno de los aspectos en que hay consenso es que las medidas de CVRS deben reflejar la percepción de las personas legas en la materia, incluidos los pacientes.

Los métodos actuales de evaluación de Calidad deVida en Relación a la Salud sehan desarrollado sobretodo a partir de trestradiciones de investigación (Angermeyer, MC.; Killian, R., 2000).

- La investigación de la felicidad, proveniente dela tradición psicológica, definida en 1953 (Jones) como un constructo psicológico posible de ser investigado. La primera investigación en los EE.UU. en 1960 (Gurin y cols.) mostró que la "felicidad y el bienestar" no podían reducirse solamente al grado de humor positivo experimentado. Estudios posteriores evidenciaron la independencia delos afectos positivos y negativos relacionados con el bienestar. Estudios como los de Costa y M c Crae (1980) yAbbey y Andrewes (1985) mostraron la relación del afecto positivo con el control interno, la tendencia a la acción, el apoyo social y la extraversión, mientras los afectosnegativos muestran mayor asociación con el estrés, la depresión y la neurosis. La demostración por parte de los psicólogos de que las respuesta subjetivas (sentimientos, deseos) podían evaluarse de modo confiable y válido, a través de tests, contribuyó a jerarquizar este campo del conocimiento y llevó al desarrollo importante de la psicometría como soporte técnico de las evaluaciones (Barge-Schaapveld DQCM, Nicolson N.A., Deslepaul PAEG. \& DeVries M W., 2000).
- La investigación en indicadores sociales, proveniente de las ciencias sociales, que se centró en los determinantes sociales y económicos del bienestar. En 1930 se realiza la primera evaluación de bienestar material (King) y en los años 50 aparece por primera vez el término calidad devida (Ordway, 1953), siendo utilizado por influyentes políticos de la época. Los estudios posteriores comenzaron a mostrar la escasa o nula relación entreindicadores objetivos de satisfacción con la viday las apreciaciones subjetivas. A partir de entonces, las líneas de investigación en el campo social divergen, desde las que continúan centrándoseen indicadores objetivosa las que se concentran en indicadores subjetivos. Dentro deesta línea, diversosinvestigadores siguen discutiendo si la satisfacción debe medirse globalmente 0 en relación a distintos ámbitos de la vida específicos (Feist, G.J.; Bodner, T.E.; Jacobs, J.F.; M iles, M . \& Tan, V., 1995). Los distintos modelos teóricos sobre necesidades humanas, desarrollados por filósofos, antropólogos, científicos sociales y políticos, incluyen, a pesar de sus diferencias teóricas las siguientes categorías de necesidades ( Angermeyer y Killian, 2000):

1. Necesidadesfisiológicas(alimentación, agua, aire, cobijo de las fuerzas de la naturaleza, etc.).

2. Necesidad de relación emocional con otras personas.

3. Necesidad de aceptación social.

4. Necesidad de realización y de sentido.

-En el área de la salud, la Organización Mundial de la Salud (OM S) fue pionera en el futuro desarrollo de la Calidad deVida Relacionada con la Salud, al definirla, ya en 1948, como "... un estado de completo bienestar físico, psíquico y social y no meramente la ausencia de enfermedad". Sin embargo, esta definición de avanzada no pasó de ser una expresión de deseos y la práctica médica así como las evaluaciones poblacionales desalud fueron alejándose cada vez más de este concepto. 
El estado de salud de una población era medido tradicionalmente por la tasa de mortalidad y esperanza de vida, a pesar de que, ya en los años 50, la tasa de mortalidad de los países desarrollados de 0 ccidentealcanzó un equilibrio, volviéndose la tasa de mortalidad una medida ineficaz para diferenciar el estado de salud delas poblaciones de estos países. Por otro lado, la mayor prevalencia de enfermedades crónicas, como consecuencia de la disminución o eliminación delas enfermedades infecciosas, así como el desarrollo de tecnologías médicas que atenuaban el dolor y el malestar, sin que eso implicase una prolongación de la vida, hicieron necesaria la aparición deotras medidas de resultados más sensibles.

A comienzos de los años 80 aparece un desarrollo de perfiles de salud (Perfil de Impacto dela Enfermedad (Bergner y cols. 1981); Perfil de Salud de Nottingham (Hunt y MC Ewen, 1980); SF-36 (Ware y cols., 1981). LoS economistas también hicieron aportesimportantes al destacar la importancia de la evaluación de medidas de preferencia y/o utilidad.

Las tres tradiciones han desempeñado un rol importante en la aplicación del concepto deCVRS que, sin embargo, deja aún muchos aspectos sin resolver.

Muchos investigadores toman un enfoque operativo y sugieren que sus instrumentos miden el constructo deCalidad deVida, aunquelo más habitual es que en realidad midan algún aspecto de la capacidad funcional del sujeto, o de lo que siente o prefiere -Perfiles de Salud, Indice de Katz, M edidas de Bienestar Psicológico, que son en realidad instrumentos de detección de psicopatología etc. (Badia, X.; Salamero, M.; Alonso J., 2002)-. Muchos instrumentosestán más centrados en las propiedades psicométricas (validez y confiabilidad dela información recogida) queen explicitar el modelo conceptual del que parten. Todo ello genera confusión a la hora de tener una definición consensuada delaCVRS.

La Organización Mundial de la Salud retoma el tema, al crearse en 1991 un grupo multicultural de expertos que avanza en la definición de Calidad de Vida y en algunos consensos básicos que permitan ir dando a estecomplejo campo al guna unidad. Esta definición y puntos de consenso fueron la base de la creación del instrumento de Calidad de Vida de la OMS (WHOQ OL-100), que, a diferencia de otros instrumentos, parte de un marco teórico para su construcción, desarrolla el instrumento en forma simultánea en distintas culturas, utiliza metodologías cualitativas como los grupos focales, para evaluar la pertinencia para losfuturos usuarios delos aspectos incluidos en la evaluación.

La OMS define calidad de vida como la "percepción del individuo de su posición en la vida en el contexto de la cultura y sistema de valores en los que vive y en relación con sus objetivos, expectativas, estándaresy preocupaciones" (1994). El Grupo W H OQ OL estableceademás una serie de puntos, en relación a las medidas de calidad de vida relacionada con la salud, aceptadas por diversos grupos de investigadores.

\section{Puntosdeconsenso (Grupo WHOQ OL, 1995)}

Las medidas de CVRS deben ser:

1. Subjetivas: Recoger la percepción dela persona involucrada.

2. Multidimensionales: Relevar diversosaspectos de la vida del individuo, en los niveles físico, emocional, social, interpersonal etc.

3. Incluir sentimientos positivos y negativos.

4. Registrar la variabilidad en el tiempo: La edad, la etapa vital que se atraviesa (niñez, adolescencia, adultez, adulto mayor), el momento dela enfermedad quesecursa, marcan diferencias importantes en los aspectos que se valoran.

La definición de la OMS, adicionalmente, hace un aporte extremadamente valioso, al enfatizar la importancia para la auto-evaluación de los factores culturales. 
El siguiente diagrama, tomado de Sharon Wood, muestra la complejidad de la articulación de las diversas dimensiones de referen- cia, temporales y de experiencia, tan difícil como armonizar los colores del Cubo de Kuchler.

\author{
DIMENSIÓN DE REFERENCIA \\ ¿En relación a quién \\ me comparo?
}

DIMENSIÓN TEMPORAL

¿En relación a qué momento me comparo?

ÁREAS DE LA EXPERIENCIA

¿En qué niveles experimento lo que percibo?

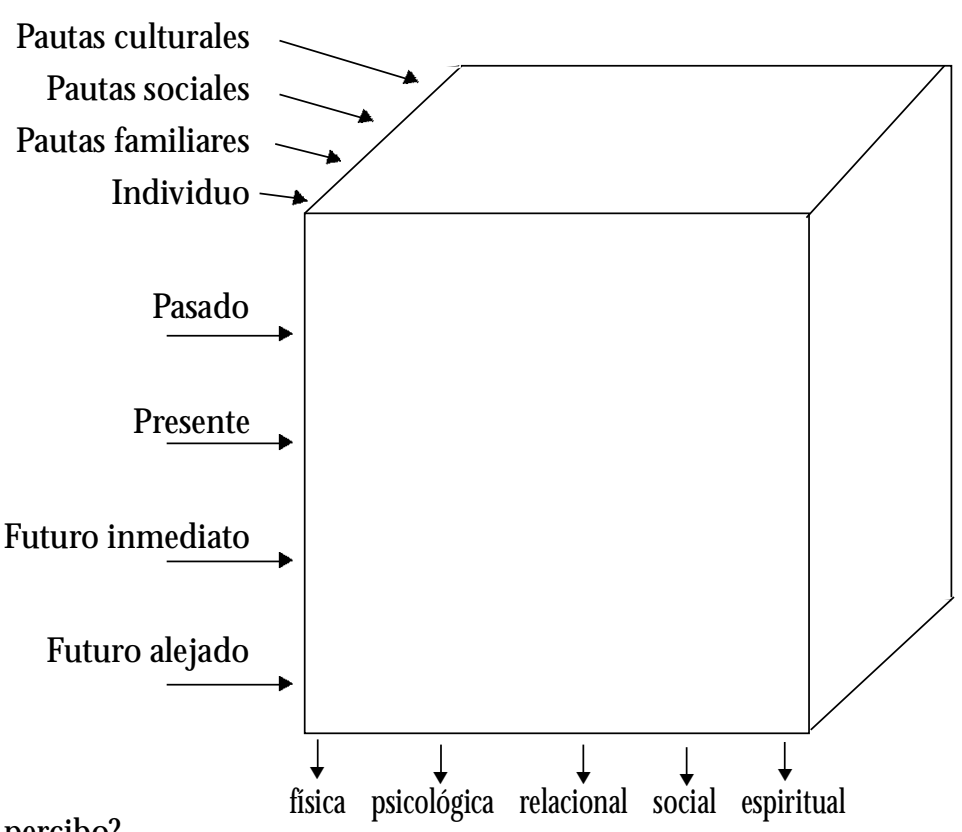

Figura 1. Dimensiones en calidad de vida.

Un excelente artículo brasileño (M inayo, Hartz, Buss, 2000) señala la relatividad del concepto de Calidad de Vida, que si bien en última instancia remiteal plano individual (es cómo la persona siente y se ve a sí misma, independientemente de queesta evaluación sea o no compartida por los demás) tiene, al menos, 3 marcos de referencia:

1. Histórico: Cada sociedad tiene parámetros diferentes de calidad de vida en distintos momentos históricos.

2. Cultural: Los distintos pueblos tienen parámetros diferentes en virtud de sus tradicio- nes culturales, a partir de las cuales se van construyendo los valores y las necesidades. El estudio transcultural de la OMS, durante el desarrollo del instrumento de evaluación de Calidad deVida (WHOQOL-100), mostró el distinto valor cultural atribuido a mantener un nivel de independencia física entre personas de EE.UU. donde la importancia de la autonomía personal, de valerse por sí mismo y no depender denadie, es un valor en sí mismo, en relación a países orientales donde tal concepción sería considerada "egoísta", pero dondeel auto-validismo asegura el no ser una carga para los demás (WHOQOL Group 1995, Szabo, 1997). I gualmente los estudios 
clásicos sobre la percepción del dolor en distintas grupos en los EE.UU. mostraron una mayor toleranciaal dolor en losanglosajones, con respecto a italianos y judíos, donde culturalmente está más "permitido" expresar el dolor físico y psíquico. Igualmente los estados de "trance" son aceptados en comunidades del Africa, no atribuyéndoles un sentido patológico, así como algunas comunidades rurales, en distintas partes del mundo, tienen una mayor tendencia a aceptar al esquizofré nico como parte de su ecosistema (Kuyken et al., 1994).

Los instrumentos de evaluación de CVRS deben combinar características que le den sustento conceptual, confiabilidad y validez (que midan lo que realmente dicen medir), pero es fundamental además que sean relevantes culturalmente, es decir que pregunten sobre las cosas que realmente le importan a las personas en un lugar determinado y en un momento histórico dado. Un trabajo realizado en Inglaterra en pacientes con SIDA (Hunt, 1998) indicó la presencia de prioridades muy diferentes en relación a salud de las que se incluyen habitualmente en los cuestionarios. Por ejemplo, se señaló la necesidad de amar y ser amado, la capacidad de disfrutar una broma, vivir libre de discriminación, poder hacer arreglos financieros, emocionales y espirituales en relación a la propia muerte, tener suficientedinero para satisfacer susnecesidades, disfrutar de la música, la naturaleza etc.

3. Clase social a la que se pertenece: Las expectativas que cada uno tiene en relación a su propia vida tienen una estrecha relación con la clase social a la que se pertenezca.

Este aspecto merece una reflexión aparte que muestra que tanto los indicadores subjetivos (percepción), como los llamados "objetivos" (ingresos, empleo, oportunidades de acceso a la salud) son igualmente valiosos, dependiendo del objetivo de la evaluación. Cuando nos referimos exclusivamente al individuo, y aún más específicamenteal individuo viviendo con su enfermedad, es válido evaluar la percepción de su nivel de bienestar o malestar con la situación quetieney tomarlo como base para decisiones significativas. Pero, cuando estamos pensando en términossociales más amplios o de políticas de Estado, no sería ético considerar que si alguien que vive en la pobreza está satisfecho con su situación en la vida (porque no tiene mayores expectativas, porque nunca conoció otra realidad), la sociedad en su conjunto y el Estado en particular no sean responsables de la modificación de esa situación. Este criterio es recogido en el ÍndicedeD esarrollo Humano (IDH ), adoptado por el PNUD (Programa deNaciones Unidas para el Desarrollo), que incluye en su determinación la renta per cápita, la salud, evaluada por la esperanza de vida al nacer y la educación, considerando la tasa de alfabetización de adultos y la matrícula en educación primaria, secundaria y terciaria.

\section{HACIA UN MODELO DE EVALUA- CIÓN DE FACTORES PSICOSOCIALES DETERMINANTES DE LA CALIDAD DE VIDA RELACIONADA CON LA SALUD}

Para concluir proponemos un modelo que intenta avanzar en la comprensión de algunos determinantes en los resultados en la CVRS, que permita ser probado empíricamente y produzca las evidencias que justifiquen la inclusión de intervenciones dirigidas a impulsar el bienestar psicosocial, además de las acciones en el campo biomédico, indudablemente imprescindibles.

La investigación reciente en psico-neuroinmuno endocrinología comienza a sentar bases empíricas queestablecen relaciones entreestado de ánimo einmunidad y a su vez la influencia sobre el estado de ánimo de la esperanza, las "ganas de vivir" o, inversamente, el desaliento, la pérdida de sentido, la soledad. Desdeel punto de vista clínico, es clásico observar que, para muchos pacientes sus valores personales o sus creencias religiosas, ideológicas, políticas, influyen sobre su esta- 
do de ánimo y pueden ser una fuente de fortaleza queinfluye sobrela salud. La necesidad de darle un sentido a la vida es un rasgo universal, quees esencial a la vida misma y la falta de sentido puede engendrar sentimientos de vacío y desesperación (¿Para qué vivir? ¿Cuál es el sentido?). La necesidad de esperanza y voluntad de vivir es importantetanto para personas sanas como enfermas. Se ha demostrado quepara los pacientes la esperanza y la voluntad de vivir son factores importantes en el proceso de recuperación. Para muchas personas la fe en sí mismos, en los otros o en un ser superior contribuye a dar sentido a la vida y puede tener influencia sobre el nivel de esperanza y el deseo de vivir. Con el avancedela investigación psico-neuroinmuno-endócrina comenzarán a comprenderse mejor los mediadores fisiológicos que explican el papel de los sucesos vitales estre santes y el soporte social, en la salud y en la calidad de vida percibida (Ross, 1994; Hytko, J.; Kright, S., 1999; O'Connel, K.; Lofty, M.; Fleck, M.; M engech, A.; Eisemann, M.; Elbi, H.; Schwartzmann, L., 1999; Brady, M.J.;
Peterman, A.H.; Fitchett, G.; Mo, M.; Cella, D., 1999; Shosharah, F.; M aly, R., 1999).

En nuestros trabajos de investigación en el Departamento dePsicología M édica dela Facultad de M edicina, consideramos la calidad de vida como un proceso dinámico y cambiante que incluye interacciones continuas entre el paciente y su medio ambiente. De acuerdo a este concepto, la calidad de vida en una persona enferma es la resultante dela interacción del tipo de enfermedad y su evolución, la personalidad del paciente, el grado de cambio queinevitablementese produceen su vida, el soporte social recibido y percibido y la etapa de la vida en que se produce la enfermedad (Schwartzmann, L.; O laizola, I.; Guerra, A.; Dergazarian, S.; Francolino, C.; Porley, G.; Ceretti, T., 1999).

Dicho resultado se mide en la percepción del grado de bienestar físico, psíquico y social y en la evaluación general de la vida que hace el paciente, teniendo en cuenta los cambios que estas situaciones pueden producir en el sistema de valores, creencias y expectativas.

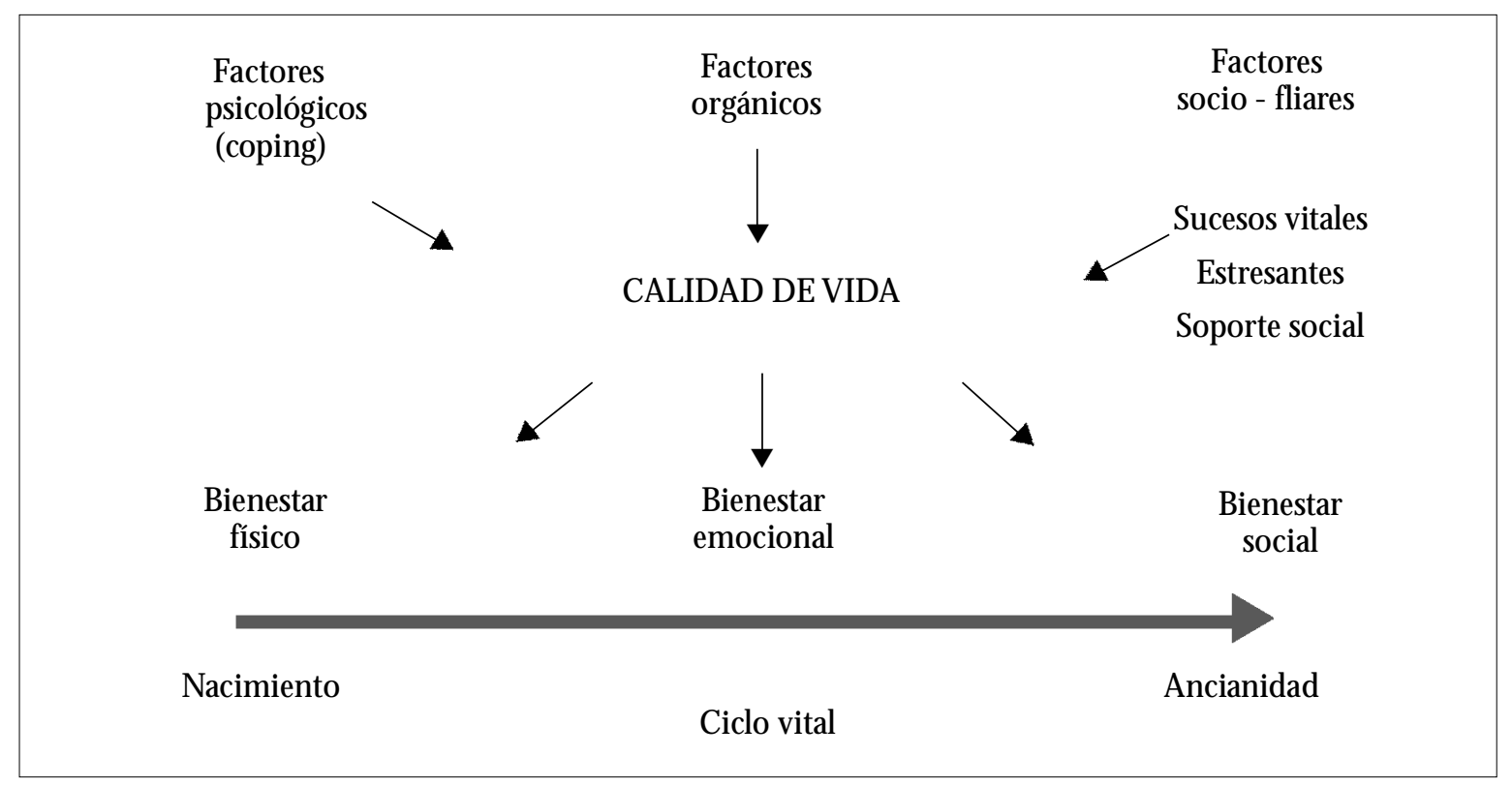

Figura 2. Cal idad de vida relacionada con la salud. 
Los procesos de adaptación a las situaciones van llevando igualmente a cambios en la valoración, los patrones temporales o interpersonales en relación a los cuales se realiza la evaluación son también cambiantes. Esta característica humana nos la recuerda Lewis Carroll en su famoso libro Alicia en el país de las maravillas: "Sabía quién era cuándo me levantéen la mañana, pero desde entonces he cambiado muchas veces".

Desdeel punto devista clínico es entonces posible intentar probar la relación entre el proceso de adaptación a la enfermedad, estudiando los mecanismos de afrontamiento (coping), así como los patrones en relación a los cuales la persona se compara y los valores y expectativas en relación a sus logros personales, modificados o no por el proceso de adaptación. La calidad de vida resultante dependería de la medida en que los mecanismos de afrontamiento y la adecuación de las expectativas permitiera que la brecha entre expectativas y percepción de la situación actual no fuese tan desproporcionada. En este mismo sentido actuaría el soporte social percibido, mientras que la acumulación de sucesos vitales que producen estrés, además de la propia enfermedad, incidirían negativamente (pobreza, desempleo, duelos, etc.).

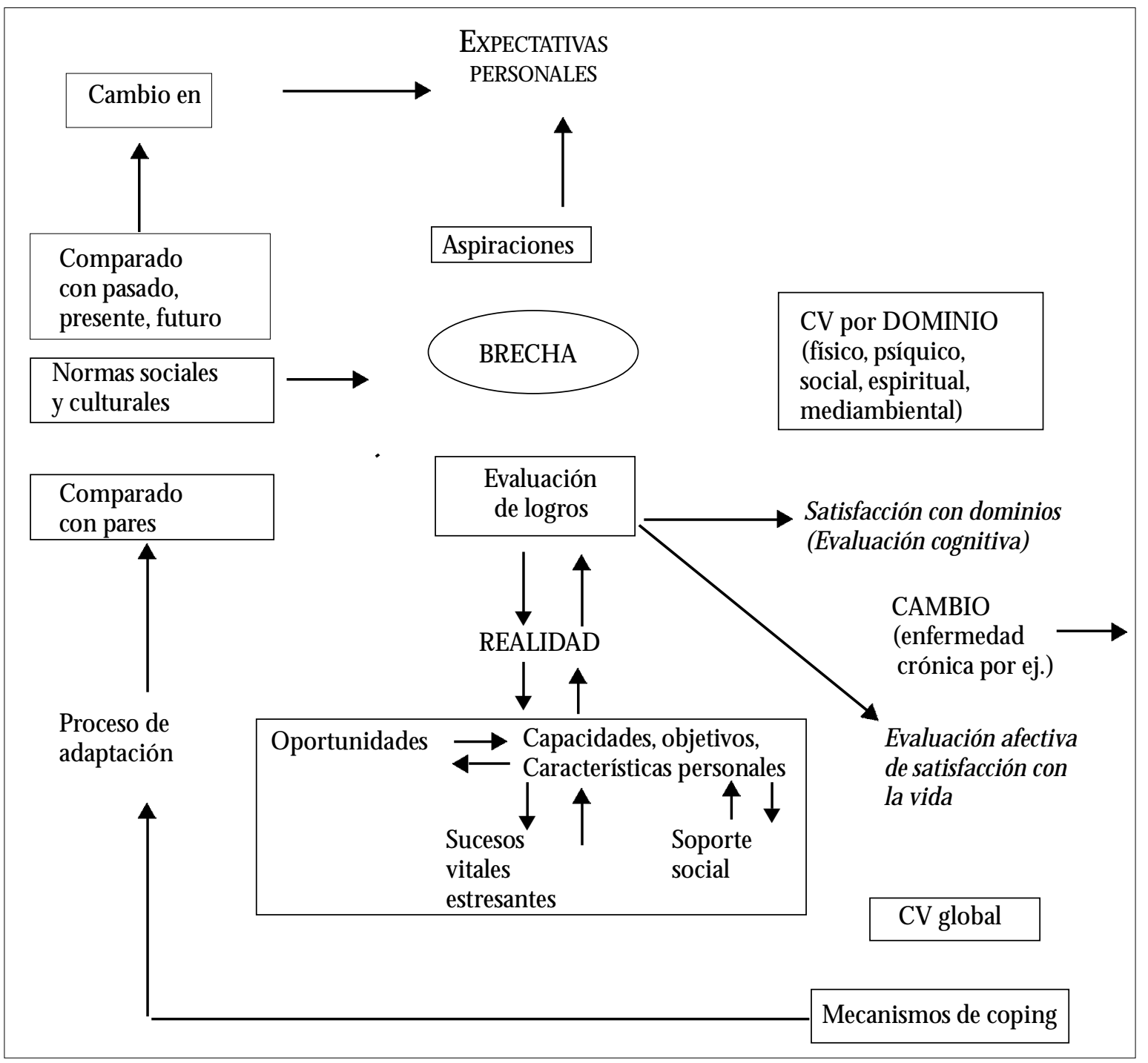

Figura 3. M odelo de calidad de vida (Adaptado de Kuok Fai Leung, 2002). 
La verificación empírica de este modelo permitiría contar con la evidencia científica para pasar del modelo biomédico que apunta fundamentalmente a los aspectos biológicosdela enfermedad (signos, síntomas) a un modelo integral bio-psico-social que, además de tomar en cuenta todos los aspectos clínicos relevantes, basados en la mejor evidencia disponible, agregue la consideración de los factores psicosociales, a través de intervenciones que, apuntando a mejorar el soporte social y flexibilizar mecanismos de afrontamiento, permitan promover la mejor calidad de vida posible, recordando la frase de Bacon con la que comenzamos esteartículo: "El oficio de la medicina no es sino tocar la curiosa arpa que es el cuerpo humano y restituirlesu armonía".

\section{REFERENCIAS BIBLIOGRÁFICAS}

AN GERM EYER, M C.; Killian, R. (2000). Modelosteóricos de Calidad de Vida en trastornos mentales. En: Katschnig, H.; Freeman, H.; Sartorious, N., Calidad de vida en los trastornosmentales, 19-29. Masson, Barcelona.

BADIA, X.; Salamero, M.; Alonso, J. (2002). La medida de la salud: guías de medición en español. Edimac, Barcelona.

BARGE-SCHAAPVELD, DQCM ., Nicolson, NA., Deslepaul, PAEG. \& De Vries, M.W. (2000). Evaluación de la calidad de vida diaria con el método de muestreo de experiencias. En: Katschnig, $\mathrm{H}$.; Freeman, H.; Sartorious, N ., Calidad de vida en los trastornos mentales, 93-105. Masson, Barcelona.

BERGNER, M.; Bobbit R.A.; Carter, W.B.; Gibson, B.S. (1981). The Sickness Impact Profile: Development and final revision of a health status measure. $M$ ed Care, 19:787-805).

BONICATTO, S.; Soria J. J. (1998). WHOQOL, LOS Instrumentos de Calidad de Vida de la Organización Mundial dela Salud. M anual del Usuario. La Plata, CATA.

BRADY, M.J.; Peterman, A.H.; Fitchett, G.; Mo, M.; Cella, D. (1999). Case for including spirituality in quality of life measurement in oncology. PsychoOncology 8: 417-428.

CARRASCO, R. L. (1998). Versión española del WHOQOL. Madrid, Ergon.
DOCHERTY, J.P. ; Streeter, M.J. (1996). M easuring outcomes. En: Outcomes assessment in clinical practice. Williams \& Wilkins, Baltimore, M aryland, USA, pp. 8-18.

FEIST, G.J.; Bodner, T.E.; Jacobs, J.F.; M iles, M . \& Tan, V. (1995). Integrating top-down and bottom up structural models of subjective well lbeing: A longitudinal investigation. J. Personality Soc. Psychol., 68, M S 50-M S 56.

GURIN, G; Veroff, S; Field S. (1960). Americans view their M ental Health. Basic Books, N ew York.

GUYATT, G.H.; Feeny, D.H.; Patrick, D. (1993). M easuring Health-Related Quality of Life. Annals of Internal M edicine, vol. 118 (8): 622-629.

HUNT, S.; Mc Ewen, J.; Mc Kenna S.P. (1986). $M$ easuring health status. London, $\mathrm{Croom} \mathrm{Helm}$.

HUNT, S. (1998). Cross-cultural issues in the use of quality of life measures in randomized controlled studies. En: Quality of Life Assessment in Clinical Trials. Ed. Staquet, M.; H ays, R.; Fayers, P.M.; Oxford University Press, pp. 51-68.

HYTKO, J.; Kright, S. (1999). Body, mind and spirit: towards the integration of religiosity and spirituality in cancer quality of life research. Psycho-oncology 8: 439-450.

JONES,H M (1953). The pursuit of happiness. Harvard University Press, Cambridge, M A

KUYKEN, W.; Orley, J.; Hudelson, P.; Sartorius, S. (1994). Quality of LifeAssessments acrosscultures. International Journal of M ental Health, 23 (2): 527.

LEPLÈGE, A. y Hunt, S. (1998). El problema de la Calidad de Vida en M edicina, Jama (ed. esp.), vol. 7, № 1: 19-23.

LEUNG, K.F. (2002). A Systemic model for HealthRelated Quality of Life. Newsletter for ISOQOL M embers. Vol. 7, 3: 2-3.

LEVI, L. (2001). Psycho-socio-economic determinants for stress and depression: A call for action. En: Coping with stress and depression related problems in Europe. Final Report European Union Presidency, Brussels, 25-27/10/2001: 17-20.

M INAYO, M.C., Hartz, Z.M ., Buss, P. (2000). Quality of life and $\mathrm{H}$ ealth: a necessary debate. Ciencia $\&$ Saúde Coletiva. ABRASCO, Vol. 5, 1, 7-18.

NAUGHTON, M .J.; Shumaker, S.A.; Anderson R.T.; Czajkowski, S.M . (1996). Psychological Aspects of Health-Related Q uality of LifeM easurement: Tests and Scales. En Quality of Life and Pharmaco economics in Clinical Trials. Spilker, B. Cap. 15 , 117131, N ew York, Lippincott-Raven.

O'CON NEL, K.; Lofty, M.; Fleck, M.; Mengech, A.; Eisemann, M.; Elbi, H.; Schwartzmann, L. (WHOQOL GROUP) (1999). How do spiritual, religious and personal health beliefs affect quality 
of life: further development into theWorld H ealth Organization (WHOQOL-100) measure. Quality of Life Research; Vol. 8: 606.

PATRICK, D., Erickson P. (1993). Health Policy, Quality of Life: Health Care Evaluation and Resource Allocation. Oxford University Press. N ew York.

ROSS, L. (1994). Spiritual Aspects of Nursing. J-AdvNurs., 1994: 19(3): 43.

SCH WARTZM AN N, L.; Olaizola,l.; Guerra A.; Dergazarian, S.; Francolino C.; Porley,G.; Ceretti, T. (1999). Validación de un instrumento para medir calidad de vida en Hemodiálisis crónica: Perfil de impacto de la enfermedad. Revista M édica del U ruguay. Vol.15, №2. Agosto: 103-109.

SH OSH ARAH , F.; M aly, R. (1999): Coping with breast cancer in later life: the roleof religious faith. Psychooncology 8: 408-416

SZABO, S. (1996). The World Health Organization Quality of Life (WHOQOL) Assessment Instru- ment. En Q uality of Life and Pharmaco-economics in Clinical Trials. Spilker, B. (III: 36) (355-362) New York, Lippincott-Raven.

TESTA M . (1996). Current Concepts: Assessment of Quality-of-Life Outcomes. N Engl J Med, Volume 334(13). M arch 28, 835-840.

WARE, (1996). The SF-36 Health Survey. En Quality of Life and Pharmacoeconomics in Clinical Trials. Spilker, B. (III:34) (337-346), New York, LippincottRaven.

WHOQOL GROUP (1995). TheWorld Health Organization Quality of life assessment (WHOQ OL). Position Paper from the World Health Organization. Soc. Sci. M ed. Vol. 41, № 10, pp. 1.403-1.409.

WOOD, S. (2001). Taller deIntroducción al estudio de la Calidad de Vida Relacionada con la Salud. 1a. Reunión Latinoamericana de Calidad de Vida,1617 agosto, Montevideo, Uruguay (comunicación personal). 